\title{
Viajeros alemanes en Alta Verapaz en el siglo XIX. Su aportación al conocimiento de las lenguas y cultura mayas
}

\author{
German travelers in Alta Verapaz during the 19th century: \\ Their contribution to the study of Mayan languages and culture
}

Laura Caso Barrera ${ }^{1}$

\section{Resumen}

En este trabajo se analizará de manera sucinta la vida y obra de tres viajeros alemanes que llegaron en el siglo XIX a Guatemala: Erwin Paul Dieseldorff (1898-1945), Karl Sapper (1866-1945) y Karl Hermann Berendt (1817-1878). Dieseldorff y Sapper hicieron extensos recorridos por el área maya recopilando, describiendo y comparando las diversas lenguas mayenses. Dieseldorff fue un estudioso de la lengua kekchí y recuperó importantes documentos coloniales escritos en esta lengua. Por su parte, Berendt no sólo recopiló incontables cuadernos de notas sobre diversas lenguas mayas, sino que además realizó copias manuscritas de cientos de documentos coloniales escritos en lenguas indígenas de Mesoamérica. La aportación de estos tres investigadores al conocimiento de la lingüística e historia mayas es incomparable.

Palabras clave: Viajeros alemanes. Lenguas mayas. Kekchí. Alta Verapaz.

\begin{abstract}
In this paper we briefly analyze the biography and works of three German travelers who arrived in Guatemala in the XIX century: Erwin Paul Dieseldorff (1898-1945), Karl Sapper (1866-1945) and Karl Hermann Berendt (1817-1878). Dieseldorff and Sapper made extensive expeditions throughout the Maya area, collecting, describing and comparing the various Mayan languages. Dieseldorff was a scholar of the Kekchí language and recovered important colonial documents written in this language. For its part, Berendt not only collected countless notebooks on various Mayan languages, but also made handwritten copies of hundreds of colonial documents written in several indigenous languages of Mesoamerica. The contribution of these three scholars to the knowledge of Mayan history and linguistics is incomparable.
\end{abstract}

Key words: German travelers, Maya languages, Kekchí, Alta Verapaz.

\footnotetext{
${ }^{1}$ Profesora-Investigadora del Colegio de Postgraduados - Campus Puebla, México.
} 


\section{Introducción ${ }^{2}$}

Los alemanes llegaron a Guatemala desde mediados del siglo XIX, atraídos por las nuevas políticas establecidas por el gobierno guatemalteco para promover nuevos cultivos comerciales. Los productos de mayor importancia habían sido el añil y la cochinilla, utilizados como tintes en la industria textil. La cochinilla fue el producto comercial más importante de Guatemala hasta 1879 (King, 1974:91). El gobierno guatemalteco que buscaba la diversificación productiva en 1835, empezó a ofrecer incentivos económicos a quienes pudieran producir cien quintales de café. En realidad, el cultivo comercial de café no despego sino hasta 1860. El Consulado de Comercio de Guatemala, por medio de uno de sus miembros, Julio Rosignon, demostraba la importancia del Departamento de la Verapaz para diversos cultivos de importancia comercial, en particular el café. Además, esta región ofrecía la ventaja de tener rutas comerciales por la costa norte, lo que hacía más fácil transportar el café a los mercados europeos (King, 1974:92, Rosignon, 1884). Poco a poco arribaron comerciantes ingleses, belgas y alemanes a Guatemala, que con sus actividades comerciales se hicieron de un patrimonio, lo cual les permitió invertir en tierras que destinarían al cultivo de café. Muchos de los primeros comerciantes alemanes fueron trayendo a sus familias, ampliando sus posesiones y sus cultivos.

En 1877, el presidente Justo Rufino Barrios vio en la expansión del café la base financiera que permitiría la estabilidad del país. Esto promovía la entrada de capitales extranjeros vitales para la economía guatemalteca.

La población indígena suponía un obstáculo para este "progreso", por lo que Barrios abolió la propiedad comunal indígena, para que esas tierras pudieran ser compradas por los inversionistas extranjeros. Asimismo, a través de la Ley de Trabajadores del 3 de abril de 1877 se estableció el mandamiento gracias a lo cual el gobierno de Guatemala autorizaba a los dueños de estancias un pago ínfimo a los indígenas que trabajaran en sus fincas cafetaleras. La expropiación de las tierras comunales indígenas y las leyes que garantizaban mano de obra casi gratuita crearon las bases para el desarrollo del café en esta región. En el mismo año Alta Verapaz se estableció como departamento y la ciudad de Cobán se convirtió en la cabecera departamental (King 1974: 30, Wichmann y Hull 2009:873). Las principales poblaciones indígenas de Alta Verapaz eran kekchíes y pokomchíes. ${ }^{3}$ Estas poblaciones indígenas reaccionaron en forma

\footnotetext{
${ }^{2}$ Deseo agradecer a la Lic. Ana Lucía Ortiz de la Universidad Francisco Marroquín en Guatemala, haberme proporcionado una copia digital y el permiso para reproducir el mapa sobre lenguas y tribus de Alta Verapaz de Karl Sapper, que ilustra este trabajo. El original se encuentra en la mapoteca de la Biblioteca Ludwig von Mises de la Universidad Francisco Marroquín.

${ }^{3}$ La Academia de Lenguas Mayas de Guatemala escribe actualmente estos nombres como q'eqchi'y poqomchi', sin embargo en este artículo utilizó la ortografía hecha por Otto Stoll
} 
violenta ante el despojo e injusticias de las que eran objeto. En 1864 se desató una rebelión indígena liderada por Melchor Yat indígena kekchí de San Pedro Carchá, después de esta rebelión que no tuvo mayor éxito, muchos kekchíes decidieron migrar hacia Honduras Británica (actual Belice) (King 1974: 29, Thompson 1930:35). Actualmente existen comunidades kekchíes asentadas desde finales del siglo XIX en el Distrito de Toledo en Belice.

En este contexto arribaron diversas familias alemanas como la de los Dieseldorff y Sapper. En 1866 James S. Sanborn y su hija Helen, de la Compañía Cafetera Chase y Sanborn, hicieron un recorrido por Verapaz buscando hacer negocios con los finqueros alemanes. Helen Sanborn describía a los finqueros y comerciantes alemanes como "maravillosos lingüistas" que, además de su propia lengua, hablaban inglés, español, francés e italiano (Beaudry-Corbett y Hardy 2000:4). Debemos agregar que los alemanes y otros europeos asentados en Alta Verapaz se vieron obligados a aprender las lenguas indígenas de sus trabajadores, por lo que la gran mayoría hablaba fluidamente pokomchí y kekchí. Ambas lenguas pertenecen a la rama kiché de la familia lingüística maya. Actualmente existen entre 360.000 y 400.000 hablantes de kekchí en 23 municipios en Guatemala (Wichmann y Hull 2009:873). Además de hablar la lengua, muchos investigadores alemanes lograron hacer descripciones gramaticales y diccionarios.

Cuando Alta Verapaz se ligó a Europa y a Estados Unidos por lazos comerciales y económicos, también se convirtió en objeto de interés científico para muchos de los inmigrantes de origen alemán. Los estudios americanistas tenían ya una tradición en Europa lo que condujo a la creación del Congreso Internacional de Americanistas (ICA por sus siglas en inglés) en 1875 por la Sociedad Americanista francesa. El objetivo del congreso era promover los estudios etnográficos, históricos y lingüísticos sobre América principalmente antes de la llegada de los europeos. Al principio se evitó abordar el período postcontacto por temor a entrar en controversias políticas, pero conforme fueron sucediéndose los congresos, se dio una mayor apertura. Entre 1877 a 1976 los temas que abordó el congreso pertenecen al campo de la antropología, la etnología, la arqueología y la lingüística (Beaudry-Corbett y Hardy 2000:5). Los intelectuales alemanes, que además eran comerciantes y finqueros, tuvieron la oportunidad de hacer viajes y recorridos por casi toda Centro América y particularmente en Verapaz, donde llevaron a cabo importantes investigaciones arqueológicas, etnográficas, históricas, lingüísticas y epigráficas, mismas que son fuentes de primera mano para entender a los grupos mayas que abordaron. Estos investigadores fueron los primeros en señalar que existía una continuidad entre las grandes civilizaciones mayas, sus logros científicos y su escritura, con

(Etnografía de Guatemala, 1958. Seminario de Integración Social Guatemalteca. Editorial del Ministerio de Educación Pública, Guatemala, Centroamérica) que es la que utilizan los investigadores alemanes aquí analizados. 
los grupos mayas que habitaban diversas regiones de México y Centro América. Esto los llevó a rescatar y coleccionar documentos históricos hechos en el periodo colonial en lenguas indígenas, comparándolos con estudios lingüísticos y etnográficos. Su interés por descifrar la escritura jeroglífica maya los llevó a un estudio exhaustivo de documentos históricos en lenguas indígenas y a realizar estudios etnográficos y lingüísticos, que se han convertido en fuentes de insuperable valor para los científicos actuales.

Erwin Paul Dieseldorff (1868-1940), nativo de Hamburgo, fue uno de los más influyentes y ricos productores de café y comerciante en la zona de Alta Verapaz, Guatemala a principios del siglo XX. Antes de migrar a Guatemala en 1888 había recibido entrenamiento en comercio internacional en la firma de su tío C. W. Dieseldorff en Londres. En Centro América comenzó su carrera trabajando para otro tío H.R. Dieseldorff que tenía operaciones mercantiles en la ciudad de Cobán, capital de Alta Verapaz. Su tío importaba artículos de Inglaterra que comerciaba en Cobán. Al mismo tiempo Erwin P. Dieseldorff hacía trabajo voluntario en diversas fincas cafetaleras donde aprendió el cultivo y cuidado de los cafetales. Dieseldorff por medio de diversas herencias y compras, pronto se convirtió en uno de los mayores propietarios de tierras en Verapaz. Alrededor de 1897, la familia Dieseldorff, que incluía a varios hermanos y sobrinos, contaba con 52.000 acres de tierra y con 600.000 árboles de café y diversos beneficios. Dieseldorff continuó expandiendo sus posesiones comprando diversas propiedades a comunidades indígenas, por lo que él y su familia fueron de los pocos alemanes que escaparon a la expropiación de sus tierras por el gobierno guatemalteco durante la segunda guerra mundial (Adam 2005:276).

Además de sus actividades como productor cafetalero y empresario, Erwin Dieseldorff tenía gran interés en la ciencia y la arqueología. Durante sus primeros años de estancia en Guatemala acompaño al geógrafo Karl Sapper en varias expediciones a diversas partes de Centro América. Estos viajes fortalecieron su interés en la botánica local, la arqueología, la historia y las lenguas indígenas, principalmente el kekchí. Colectó y estudió plantas medicinales utilizadas por los indígenas de Alta Verapaz y trató de comercializarlas por su gran valor farmacológico (Adam 2005:277). Publicó en Alemania entre 1926 y 1933 tres volúmenes sobre la historia y religión de las antiguas civilizaciones mayas. Asimismo escribió varios artículos sobre arqueología maya y presentó varios de estos trabajos en el Congreso Internacional de Americanistas. Sus papeles científicos, personales y de negocios se encuentran actualmente en la Colección Dieseldorff de la Biblioteca Latinoamericana de la Universidad de Tulane. Son especialmente interesantes los documentos históricos escritos en lengua kekchí que Dieseldorff se dio a la tarea de coleccionar, como una copia de la Leyenda dorada de mediados del siglo XVI, un testamento en kekchí de 1539, así como títulos de tierras de varias comunidades kekchíes, textos de danzas-dramas, 
diccionarios como el recopilado por Pablo Wirsing, en alemán y kekchí (Náñez Falcón, s/f).

Asimismo la colección contiene la correspondencia personal de Dieseldorff con diversos miembros de su familia así como su correspondencia de negocios. También se encuentran cartas de este personaje con los más destacados científicos de su época, como arqueólogos, lingüistas e historiadores. Se encuentra una nutrida comunicación entre Dieseldorff y renombrados científicos como Hermann Beyer, Frans Blom, Mary Butler, Alfonso Caso, Thomas Gann, William Gates, A. V. Kidder, Walter Lehmann, F. W. Putnam, S. K. Lothrop, Teobart Maler, Enrique Juan Palacios, Juvenal Valerio Rodríguez, el padre Ildefonso H. Rossbach, Karl Sapper, Paul Schellhas, Herbert J. Spinden, Julio C. Tello, J. Eric Thompson, Alfred M. Tozzer, Max Uhle, George C. Vaillant y Luis E. Valcarcel. La gran mayoría de estos personajes conocía personalmente a Dieseldorff. Muchos eran arqueólogos que realizaban excavaciones en México y Centro América, y en las misivas describen sus trabajos y descubrimientos. Las cartas de Dieseldorff de la década de 1930 se centran en su interés por el sistema calendárico de los mayas, tema que lo obsesionaba y que compartía con otros investigadores (Náñez Falcón, s/f).

Con respecto a su interés por recopilar documentos históricos escritos en kekchí, resulta interesante la carta que escribió a Frederic Ward Putnam, curador del Museo Peabody de Arqueología y Etnología en la Universidad de Harvard:

In my hunt after old papers, now unfortunately at an end, as I do not know of more likely hiding places, I have come across a testament of the widow of a certain Luis Caal Hernández, which is interesting as it reproduces, in a more or less correct form the Indian language Kekchí, as it was spoken in the times directly following the Conquest [1583]. The Kekchí spoken at that time is just the same as spoken today; the only difference being a leaning to the accent spoken to this day in Cahabón, yet this may be accounted for by the possibility of the person making the will, or the Secretary having come from that district. The writing is often a very bad representation of the phonetic value, yet, as before mentioned, this can be easily understood, as there are no books written in Kekchí. The document being a unique one, I send it to you, as you might like to give a photographic representation of it, and after you have used it, I beg you to return it. Dr. Stoll photographed it, with my permission, but I do not think that he understood a word of it. I could formerly not make it out either, but with more perfect knowledge of the Kekchí language acquired by constant practice and with the kind help of an intelligent native, the meaning has become clear (Tozzer Library C.A 6D566F).

Dieseldorff transcribió y tradujo un testamento kekchí de 1583 y se lo envío en 1903 a Putnam, esperando que pudiera ser publicado en la revista 
American Anthropologist; Frans Boas revisó el manuscrito y consideró que su contenido era trivial y decidió no publicarlo (Tozzer Library C.A 6D566F, Weeks 1997: 64). Actualmente sabemos que el contenido del documento es de gran importancia, ya que muestra que poco tiempo después de la llegada de los españoles, los kekchíes utilizaban el alfabeto latino para escribir en su propio idioma y que habían adoptado el uso del testamento europeo para heredar sus bienes. Además, es uno de los pocos documentos coloniales escritos en la variante dialectal de Cahabón. Dieseldorff, en su carta a Putnam, señala que permitió que Otto Stoll fotografiara el documento y al parecer Karl Sapper también contaba con una copia fotográfica del mismo, que aparentemente compartió con Robert Burkitt, un irlandés estudioso de las lenguas indígenas y particularmente del kekchí. Burkitt hizo un análisis y una traducción del documento perteneciente a Dieseldorff, con el título "A Kekchí Will of the Sixteenth Century", que fue publicado en 1905 en American Anthropologist (Burkitt, 1905). Tanto Dieseldorff como Burkitt concluyeron que el testamento de 1583 podía ser comprendido casi en su totalidad, con excepción de algunas palabras y frases que habían caído en desuso, pero que un hablante de kekchí de la época podía entender su contenido sin mayor problema.

Karl Theodor Sapper (1866-1945) nació en Wittislingen; obtuvo su doctorado en geografía de la Universidad de Múnich en 1888. Posteriormente viajó a Alta Verapaz, Guatemala, donde su hermano Richard Sapper era dueño de varias fincas cafetaleras. Karl se estableció en Alta Verapaz para administrar algunas de las propiedades de su hermano, quien poseía 30.000 acres de tierra y 550.000 plantas de café (Adam 2005: 934). Siendo administrador de estas propiedades, Karl Sapper hizo diversos recorridos por Alta Verapaz, donde realizó investigaciones geográficas, geológicas, etnográficas, recopilando además información histórica y lingüística. También realizó una expedición desde Tehuantepec hasta Panamá, la mayor parte a pie, en búsqueda de información científica, tanto geográfica como etnográfica. A pesar de sufrir de malaria, recorrió cientos de kilómetros de selvas tropicales, utilizando antiguos caminos y sendas, para llevar a cabo sus estudios geológicos y geográficos (McBirney y Volker 2003: 79). Trabajó como geólogo para el gobierno mexicano antes de regresar a Europa en 1900, donde se convirtió en profesor de las universidades de Tubinga, Leipzig, Estrasburgo y Würzburg (BeaudryCorbett y Hardy 2000:6). Muchas de sus investigaciones etnográficas, históricas y lingüísticas fueron presentadas en el Congreso Internacional de Americanistas, junto con los trabajos de otros reconocidos investigadores como Otto Stoll y Erwin Dieseldorff, mencionado anteriormente. Los doce años de recorridos e investigaciones de campo en Centro América dieron un auge a las investigaciones de esta región, sus poblaciones y culturas. El trabajo de Sapper dio a conocer datos etnográficos sobre diversos grupos de los que no se tenía información hasta ese momento. 
Los estudios etnográficos hechos por Sapper muestran que las poblaciones mayas que describió continuaban con sus tradiciones culturales, el uso de sus lenguas y religión, lo que daba una persistencia a su identidad e historia manteniendo su sentido de comunidad, a pesar de haber perdido sus tierras comunales para convertirse en trabajadores de las plantaciones cafetaleras. Sus mapas de la región lo llevaron a obtener, en 1893, premios en la Feria Mundial de Chicago (McBirney y Volker 2003:80). Hizo mapas topográficos y lingüísticos para el gobierno mexicano y gobiernos de Centro América. Las observaciones y trabajos arqueológicos, etnográficos y lingüísticos han permanecido como fuentes primarias para el conocimiento sobre Centro América aún hoy día.

En su trabajo "Choles y Chortíes" publicado en 1907, en el XV Congreso Internacional de Americanistas, Sapper presenta un exhaustivo trabajo histórico y etnográfico para entender la diversidad lingüística existente en Alta Verapaz. Originalmente este trabajo iba acompañado por un mapa donde se ubicaban los diferentes grupos étnicos mencionados en el documento; desgraciadamente el mapa no fue incluido en la publicación original. Sapper fue el primer investigador en señalar la existencia de grupos mayas conocidos actualmente como lacandones históricos y acaláes hablantes de lengua chol o cholchí. La Verapaz colindaba con las tierras bajas mayas del sur, donde se localizaban poblaciones mayas insumisas como eran los lacandones históricos, acaláes, choles del Manché e itzaes, estos últimos hablantes de maya yucateco (Caso Barrera y Aliphat 2012:282). Los lacandones históricos, topiltepques, acaláes y choles del Manché, eran poblaciones hablantes de lengua chol o choltí (cholchí) (según Morán 1695).

Para entender los vínculos entre las tierras bajas mayas del sur y los altos de Guatemala y Chiapas en tiempos prehispánicos y coloniales, es de gran importancia conocer las interacciones y relaciones de afinidad, intercambio, competencia y conflicto existentes entre las poblaciones kekchís de La Verapaz, con las naciones cholanas como eran los lacandones históricos, choles del Manché y los itzaes hablantes de maya yucateco, asentados en la Laguna de Petén Itzá (Caso Barrera y Aliphat 2007, 2012:282-283). El texto de Sapper es el primero en analizar fuentes coloniales y contrastar estos datos con datos etnográficos y lingüísticos de su tiempo. Como bien señala el autor, después de la llegada de los españoles a Verapaz muchas de las poblaciones de habla chol fueron reubicadas con poblaciones kekchís como lo aseguraban fuentes coloniales. Como apunta Sapper, al analizar un documento conocido como "Título del Barrio de Santa Ana", de 1565, los habitantes de este barrio de habla pocomchí establecían como su lugar de origen a Chamá, lugar que los habitantes del barrio de San Marcos en Cobán, reclamaban como parte de sus tierras originales, sin embargo el documento aclara que los de San Marcos eran acaláes (hablantes de lengua chol) que fueron reubicados por los españoles en Cobán (Sapper 2004:120-121). 
Este complejo mosaico lingüístico existente hasta el siglo XVII evidentemente había dejado una huella en las poblaciones indígenas de Alta Verapaz. La conclusión a la que llega Sapper en este artículo, es que las poblaciones de habla cholchí o choltí, reubicadas en Cobán, perdieron rápidamente su lengua y costumbres y se absorbieron a la población kekchí. Mientras que las poblaciones choles asentadas con la población kekchí en Lanquín, Cahabón y Chisec perdieron su lengua, aunque mantuvieron algunos rasgos de está, así como buena parte de su cultura (Sapper 2004:124-125). Sabemos que las poblaciones kekchíes asentadas en Lanquín y Cahabón tenían un trato constante con poblaciones choles del Manché, con quienes comerciaban e incluso parecen haber tenido fuertes lazos sociales, por lo que esta variante dialectal aún conserva influencia de la lengua cholchí (Caso Barrera y Aliphat 2007, 2012: 289-292; Feldman 1978). En su trabajo Sapper hace una interesante comparación entre vocabularios de chol de Tila y Tumbalá, Chiapas y del chortí de Chiquimula en Guatemala y Honduras, para mostrar la cercanía de estas lenguas. Sin embargo, se debe señalar que existe una confusión entre los choles que habitan en Chiapas hasta la actualidad, con las poblaciones choles del Manché de habla choltí, que debido a una reubicación forzada se extinguieron a finales del siglo XVII. Desgraciadamente, como el mismo Sapper lo menciona en su artículo, no pudo comparar estas lenguas cholanas con el diccionario choltí atribuido a fray Francisco Morán, lo que le hubiera mostrado las diferencias substanciales que existen entre estas lenguas cholanas.

Podemos apuntar que Sapper es el primero que trata de explicar las diferencias dialectales que existen en la lengua kekchí, ya que existen dos variantes, el dialecto que se habla hacia el oeste, conocido como dialecto de Cobán, que se usa en la ciudad de Cobán, San Pedro Carchá y San Juan Chamelco y la variante del este que corresponde a Lanquín, Cahabón y Senahú (Wichmann y Hull 2009: 878) (ver mapa 1). ${ }^{4}$ Actualmente sabemos que durante el período clásico en las tierras bajas mayas habitaron poblaciones de habla yucateca y chol que fueron responsables del gran desarrollo de la civilización maya. Estas poblaciones de las tierras bajas mayas, tuvieron contacto con las poblaciones kekchíes desde el postclásico y al parecer tuvieron una fuerte influencia en su lengua y cultura (Arnauld 1999, Caso Barrera y Aliphat 2007, 2012, Wichmann y Hull 2009). Como buen geógrafo Sapper fue un viajero incansable que recorrió diversas poblaciones de México y Centroamérica, realizando además estudios que se han convertido en fuentes de gran importancia para los estudios de geografía, historia, antropología y lingüística.

Karl Herman Berendt (1817-1878) nació en Danzig (Gdansk) y estudió medicina en la Universidad de Königsberg donde recibió su título en 1842.

\footnotetext{
${ }^{4}$ En el mapa titulado "La distribución de las lenguas y tribus en Alta Verapaz", Sapper señala los pueblos donde se habla kekchí, como Cobán, San Pedro Carchá, San Juan Chamelco, San Agustín Lanquín, Santa María Cahabón y Chisec.
} 


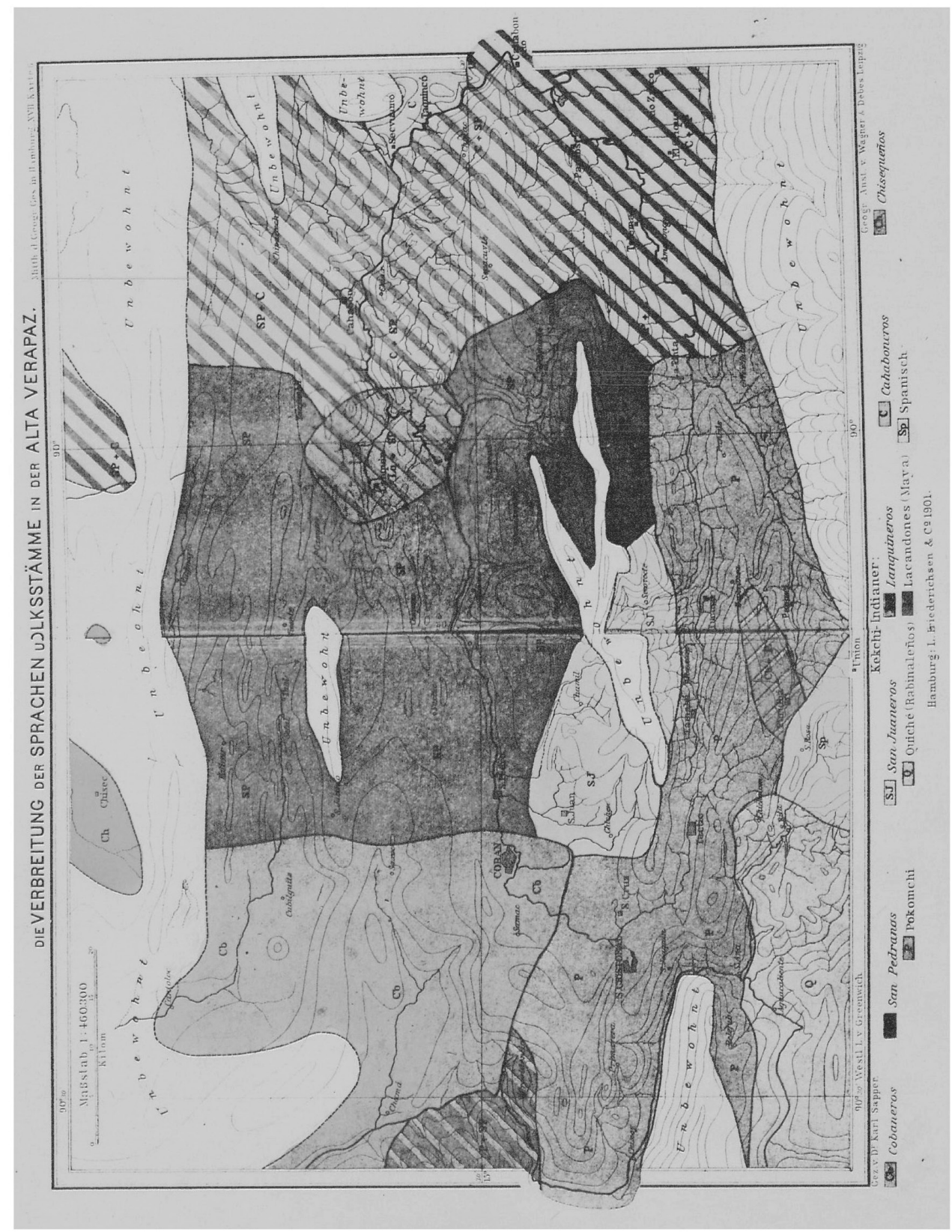

Mapa 1. Karl Sapper, Anstalt von Wagner \& Debes 1901 Die Verbreitung des Sprachen und Volksstamme in der Alta Verapaz [La distribución de las lenguas y tribus en la Alta Verapaz] (Guatemala). I. Friederchsen \& Co.: Hamburgo, Alemania.

(C) Mapoteca, Universidad Francisco Marroquín. Imagen disponible bajo la licencia de Creative Commons Reconocimiento-No Comercial-Compartir bajo la misma licencia 
Era docente de ginecología y obstetricia en la Universidad de Breslavia, pero sus ideas políticas liberales hicieron que fuera expulsado de la universidad. En 1851 decidió viajar a América donde pensó que podía expresar de manera más libre sus ideales democráticos. Llegó a Nueva York y de allí se trasladó a Nicaragua; ahí vivió dos años y empezó a aficionarse por la investigación histórica y antropológica. Posteriormente, se estableció en Veracruz en México, de 1855-1862. Pronto dejó de lado su práctica médica para dedicarse de lleno a las ciencias naturales, la etnología y la lingüística. Dedicó la mayor parte de su tiempo a viajar por los estados del sureste mexicano, Chiapas, Tabasco y Yucatán, donde recolectó especímenes de historia natural para el instituto Smithsoniano (Weeks 2002: 7-8). Parte del año 1864 lo pasó en la Biblioteca John Carter Brown de Providence, Rhode Island, transcribiendo dos importantes vocabularios mayas compilados en el período colonial. El Diccionario de Motul, en maya yucateco, atribuído a Antonio de Ciudad Real del siglo XVI y el Compendio de nombres en lengua Cakchiquel, del franciscano Pantaleón de Guzmán del siglo XVIII. Berendt podía paleografiar los documentos antiguos sin mayor problema, hablaba fluidamente el español y también hablaba maya yucateco. Logró transcribir íntegramente el Diccionario de Motul en tan sólo dos meses, pero no sólo transcribió el documento sino además hizo correcciones, añadiduras y comparaciones con otros vocabularios, por lo que se considera una obra monumental (Tozzer 1997:141).

En 1865 salió de Nueva York hacía Belice, en Honduras Británica, con la idea de realizar investigaciones geográficas y antropológicas en un recorrido que comprendía desde el Mar Caribe, pasando por Belice, el Petén en Guatemala a Chiapas en México, hasta el Océano Pacífico. En estos viajes recolecto especímenes naturales, materiales arqueológicos y realizó apuntes etnográficos y compiló datos sobre varias lenguas indígenas. Asimismo fue adquiriendo valiosos documentos coloniales en diversas lenguas mesoamericanas. En 1874 Berendt se mudó a Cobán y compró una finca cafetalera donde pasaría sus últimos años de vida. A diferencia de Dieseldorff y Sapper, que difundieron bastante sus trabajos, Berendt publicó poco. Esto se entiende pues dedicó la mayor parte de su tiempo a realizar transcripciones de manuscritos coloniales en lenguas indígenas. Se puede señalar que esta es su principal contribución, pues gracias a sus reproducciones se conocen documentos ahora desaparecidos.

Después de la Independencia de México y Centro América, con la supresión de las órdenes religiosas, las bibliotecas y archivos pertenecientes a los conventos se convirtieron en propiedad nacional, albergándolos en bibliotecas y archivos públicos. Conocedor de este proceso, en 1875 Berendt estuvo un mes en Ciudad Guatemala y visitó varios archivos y bibliotecas buscando materiales en lenguas indígenas de diversa procedencia, para realizar copias manuscritas (Weeks 2002:12). En su trabajo "Collection of historical documents in Guatemala" apuntaba la importancia de los manuscritos localizados en archivos 
y bibliotecas de Guatemala para comprender la historia prehispánica y colonial de diversos grupos indígenas (Berendt 1877:421). Lo mismo había hecho en Yucatán, donde tuvo oportunidad de copiar los manuscritos en maya yucateco pertenecientes a la colección de Pío Pérez (Tozzer 1997:147). Este personaje, Juan Pío Pérez Bermón (1798-1859), fue un sabio, filólogo e investigador de la lengua y cultura mayas, que se dio a la tarea de reunir manuscritos en lengua maya yucateca. Dejó inconcluso un diccionario de la Lengua Maya, mismo que fue completado por Berendt en 1870, gracias a lo cual fue finalmente publicado (Pío Pérez 1877:VI).

En Alta Verapaz, Berendt consiguió importantes documentos originales y también hizo copias de materiales en las lenguas pokomchí y kekchí. Los materiales en pokomchí incluyen un diccionario, sermones, confesionarios, doctrinas y pláticas. Diversos documentos originales, así como transcripciones en la colección documental de Berendt provienen del archivo parroquial de Cobán; estos documentos se encuentran escritos en pokomchí y kekchí. Se localizan sermonarios, catecismos, gramáticas, glosarios, vocabularios, oraciones, doctrinas, etc. Entre los importantes textos escritos en kekchí está un "Arte de Lengua Cakchi para Bien común" sobre la que Berendt señalaba lo siguiente:

En el archivo de la Parroquia de Cobán se halla un libro manuscrito en 4to conteniendo en 192 fojas sin numeración coherente varios escritos en Pocomchí y Kekchí copiados por Juan de Morales, maestro de fiscal de San Juan Chamelco.

En la presente Copia he seguido fielmente el texto de Morales, corrigiendo solamente lo que fue fuera de toda duda y poniendo correcciones probables pero no del todo seguras, escritas con lápiz encima de la línea. Examinando el texto palabra por palabra con ayuda de un mestizo cobanero, Pedro Torres de alguna inteligencia e instrucción, he añadido con tinta encarnada al margen las formas de palabras y dicciones como se usan hoy en esta ciudad y también anotaciones mías. En estas adiciones he usado los signos fonéticos de mi Alfabeto Analítico, publicado en Nueva York 1869, por la Sociedad Etnológica. Cobán, Noviembre 12, 1875. (Berendt Linguistic Collection, Ms. Coll. 700 Item 69).

Berendt justificaba la importancia de realizar transcripciones como una manera de conservar documentos en malas condiciones en diversos archivos, haciéndolos accesibles a otros investigadores y preservándolos para el futuro. Pero no solamente hacía transcripciones, sino que hacía un análisis detallado de los textos, incluyendo anotaciones, apuntes y, en ocasiones, correcciones. Sus transcripciones fueron utilizadas y citadas por otros investigadores como el lingüista y etnólogo suizo Otto Stoll (Weeks 2002:13). La Colección Lingüística Berendt fue adquirida por Daniel G. Brinton y actualmente se 
alberga en la Biblioteca Van Pelt de la Universidad de Pensilvania. Comprende 183 entradas pertenecientes a más de 40 lenguas indígenas de México y Centro América, pertenecientes a la familia de lenguas arawak, chibcha, chocó, huave, maya, misumalpa, oto-mangue, mixe-zoque, subtiapa-tlapaneca y uto-azteca, del siglo XVI a finales del siglo XVIII. Incluye 40 manuscritos originales, 43 transcripciones fieles de manuscritos originales, 34 monografías no publicadas, 6 transcripciones completas y algunas incompletas, algunas monografías publicadas y 60 cuadernos de notas manuscritas (Weeks 2002:13). Estos cuadernos de notas generalmente incluyen descripciones etnográficas, vocabularios, comparaciones entre varias lenguas, dibujos, etc. También tiene documentos misceláneos con anotaciones diversas y correspondencia. Sus notas de campo están escritas en alemán, junto con español y maya yucateco. En su cuaderno sobre notas en lengua maya tiene descripciones de la lengua maya que se habla en el Petén, así como anotaciones sobre la lengua de los lacandones modernos establecidos cerca del Río de la Pasión. También contiene historias y canciones escritas en lengua maya yucateca (Berendt Linguistic Collection 498.21 M.L.545). Muchas de las lenguas descritas o transcritas por Berendt ahora se encuentran moribundas o extintas, haciendo de su colección lingüística una de las más importantes. Berendt sentó las bases para los estudios sobre Mesoamérica y particularmente sobre el área maya (Weeks 1998).

\section{Consideraciones finales}

En la segunda mitad del siglo XIX Guatemala trataba de consolidarse como un Estado-Nación, transformando la producción y organización económica heredada del período colonial. Así fueron dejando de lado cultivos como la grana, el añil y el cacao que fueron sustituidos por un nuevo producto comercial: el café. Los gobiernos liberales consideraban a los indígenas como sujetos inferiores, retrógrados e incapaces de convertirse en la base de una sociedad progresista. El problema principal de esta "cuestión india" era la propiedad de la tierra, por lo que los gobiernos liberales atacaron la propiedad comunal (Caso Barrera 2001:150-151). El régimen liberal instaurado en 1871 por el presidente Justo Rufino Barrios estableció el marco legal para que los inversionistas extranjeros pudieran adquirir tierras comunales y de cofradías indígenas. Frente a la necesidad de mano de obra para trabajar en las fincas cafetaleras, el régimen liberal estableció leyes como los mandamientos (1847-1894), que consistían en el reclutamiento forzoso de la mano de obra indígena por parte del Estado. Además, se establecieron las habilitaciones por deudas (1894-1934), en las que los terratenientes enganchaban a los trabajadores indígenas por medio de deudas que debían pagar con trabajo en sus fincas (Hurtado 2008:72-73). En este contexto fue que arribaron a Guatemala inversionistas, comerciantes y expedicionarios europeos que buscaban las riquezas naturales y culturales 
que podían ser aprovechadas en Europa. A mediados del siglo XIX llegarían comerciantes alemanes y sus familias a Guatemala, para dedicarse al cultivo y la comercialización del café. La presencia alemana tuvo un fuerte impacto en las poblaciones indígenas asentadas en Alta Verapaz, que reaccionaron ante la pérdida de sus tierras y el trabajo forzado en las fincas, lo que llevó a una diáspora kekchí hacia Belice.

Entre los comerciantes alemanes que arribaron a Guatemala, hubo verdaderos científicos que se interesaron por la geografía, la botánica, así como la cultura, la historia y las lenguas de los diversos grupos étnicos mesoamericanos. Mientras que el estado guatemalteco reivindicaba el nacionalismo en la antigua civilización maya, no reconocía a los grupos étnicos vivos como herederos de la misma, pues los veía como el producto de la dominación colonial. En Guatemala y México, los intelectuales liberales intentaron crear un sentido de identidad nacional recuperando y exaltando el pasado prehispánico, como antecedente glorioso del Estado nacional que se estaba edificando (Dávila 2010:192-193, Pacheco Chávez 2001:136-137). En contraposición a esta postura de los estados guatemalteco y mexicano, Erwin P. Dieseldorff, Karl Sapper y Karl Berendt, reconocieron que los indígenas mayas continuaban con sus prácticas y tradiciones culturales, así como con la preservación de diversas lenguas mayas, lo que les daba una persistencia histórica y una identidad propia. Los tres se dieron a la tarea de recopilar documentos históricos en lenguas mayas, lo que les permitió entender la historia de varios grupos étnicos desde su pasado prehispánico hasta la dominación colonial. El hecho de que hablaran lenguas indígenas como el yucateco, kekchí y pocomchí les permitió demostrar la continuidad de los grupos indígenas en Alta Verapaz, desde el período prehispánico, ya que pudieron traducir documentos tempranos del siglo XVI escritos en estas lenguas. Es así, que los intelectuales alemanes, brevemente analizados en este trabajo, dieron una secuencia histórica a los grupos mayas vivos, conectándolos con su pasado prehispánico.

Estos intelectuales fueron incansables viajeros que recorrieron México y Centro América, compartiendo su lugar de residencia en Cobán, Alta Verapaz. A pesar de vivir en esta región alejada y de difícil acceso, los científicos alemanes aquí analizados lograron proyectar sus trabajos arqueológicos, históricos y lingüísticos en foros internacionales como el Congreso Internacional de Americanistas. Su presencia puso en el mapa a Alta Verapaz, adonde llegarían otros viajeros-intelectuales de la época, con quienes estuvieron en contacto y discusión permanente. Tal fue el caso del etnólogo y lingüista Otto Stoll, el lingüista y antropólogo Robert Burkitt, el arqueólogo Alfred C. Maudslay y el reconocido mayista Eric. P Thompson. Sus aportaciones a la arqueología, la historia y la etnología de Mesoamérica son incomparables, pero sobre todo se debe subrayar el impulso que dieron al estudio de las diversas lenguas mayas. 


\section{Referencias}

American Philosophical Society, Philadelphia

Berendt Linguistic Collection, Biblioteca Van Pelt de la Universidad de Pensilvania.

Biblioteca Ludwig von Mises, Universidad Francisco Marroquín

Latin American Library, Universidad de Tulane

Tozzer Library, Universidad de Harvard

Adam, Thomas (editor). 2005. Germany and the Americas. Culture, Politics and History. Santa Barbara, California: ABC-Clio.

Arnaud, Charlotte, Marie. 1994. "Kekchíes y Pokomchíes”. En Historia General de Guatemala. Jorge Luján M. (dir.). Guatemala: Fundación para la Cultura y el Desarrollo; tomo I, pp. 693-700.

Burkitt, Robert. 1905. "A Kekchi Will of the Sixteenth Century". American Anthropologist 7(2):271-294.

Caso Barrera, Laura. 2001. "Entre civilización y barbarie. La visión de los historiadores liberales sobre la Guerra de Castas de Yucatán”. En: México: Historia y alteridad. Perspectivas multidisciplinarias sobre la cuestión indígena. Yael Bitrán (coord.). México: Universidad Iberoamericana; pp. 149-177.

Caso Barrera, Laura y Mario Aliphat F. 2007. "Relaciones de Verapaz y las Tierras Bajas Mayas Centrales en el siglo XVII”. En: Memorias del XX Simposio de Investigaciones Arqueológicas en Guatemala. Juan Pedro Laporte, Bárbara Arroyo y Héctor Mejía (eds.). Guatemala: Ministerio de Cultura, Instituto de Antropología e Historia, Asociación Tikal y Fundación Arqueológica del Nuevo Mundo; pp. 4555 .

2012. "Mejores son huertos de cacao y achiote que minas de oro y plata: Huertos especializados de los choles del Manché y de los k'ekchi'es". Latin American Antiquity 23(3):283-300.

Berendt, Karl H. 1877. "Collections of Historical Documents in Guatemala". Annual Report of the Smithsonian Institution for 1876, Washington, D.C.; pp. 421-423.

Beaudry, Corbett Marilyin y Hardy, Ellen T. (eds.). 2000. Early Scholars' Visits to Central America. Reports by Karl Sapper, Walter Lehmann and Franz Termer. University of California, Los Angeles: Cotsen Institute of Archaeology.

Dávila, Roxanne. 2010. "Forjando una nación (1830-1839): La obra de Juan Galindo dentro del proyecto de colonización del Jefe de Estado de Guatemala Mariano Gálvez". Anales de la Academia de Geografía e Historia de Guatemala LXXXV:188-200.

Feldman, Lawrence H. 1978. "Los choles entre los kekchies". Anales de la Sociedad de Geografía e Historia de Guatemala LI:79-112.

Hurtado Paz y Paz, Laura. 2008. Dinámicas agrarias y reproducción campesina en la globalización. El caso de Alta Verapaz, 1970-2007. Guatemala: FyG Editores.

King, Arden R. 1974. Coban and the Verapaz. History and Cultural Process in Northern 
Guatemala. New Orleans: Middle American Research Institute, Tulane University.

McBirney, Alexander R. and Volker, Lorenz. 2003. "Karl Sapper: Geologist, Ethnologist and Naturalist". Earth Sciences History: Journal of the History of the Earth Sciences Society 22(1):79-89.

Morán, Francisco. 1695. Arte y vocabulario de la lengua cholti o lengua de milperos. Philadelphia: Microfilm in the American Philosophical Society.

Nañez, Falcón Guillermo. s/f. Dieseldorff, Erwin Paul, Papers. Inventory of Collection. Tulane: Manuscripts Department, Tulane University Library.

Tozzer, Alfred M. 1977. A Maya Grammar. Nueva York: Dover Publications.

Pacheco Chávez, Ma. Antonieta, Ilhui. 2001. "Manuel Larráinzar. Espacio y presencia de los indios chiapanecos en la reconstrucción de la historia nacional". En: México: Historia y alteridad. Perspectivas multidisciplinarias sobre la cuestión indígena. Yael Bitrán (coord.). México: Universidad Iberoamericana; pp. 131-148.

Pío Pérez, Juan. 1877. Diccionario de la Lengua Maya. Mérida: Imprenta Literaria de Juan F. Molina Solís.

Rosignon, Julio. 1881. Porvenir de La Verapaz en La República de Guatemala. Memoria dedicada al Consulado de Comercio de Guatemala. Guatemala: Imprenta de Luna.

Sapper, Karl. 2004. “Choles y chortíes”. Liminar. Estudios Sociales y Humanísticos, 2(4):119-142.

Stoll, Otto. 1958. Etnografía de Guatemala. Seminario de Integración Social Guatemalteca. Guatemala: Editorial del Ministerio de Educación Pública.

Thompson, Eric J. 1930. Ethnology of the Mayas of Southern and Central British Honduras. Chicago: Field Museum of Natural History of Chicago.

Weeks, John M. 1997. "Subregional Organization of the sixteenth-century Q'eqchi' Maya, Alta Verapaz, Guatemala". Revista Española de Antropología Americana 27:59-93.

. 1998. "Karl Hermann Berendt: colección de manuscritos lingüísticos de Centroamérica y Mesoamérica". Mesoamérica 36:619-693.

. 2002. The Library of Daniel Garrison Brinton. Philadelphia: University of Pennsylvania Museum of Archaeology and Anthropology.

Wichmann, Soren y Kerry Hull. 2009. "Loanwords in Q'eqchi'. A Mayan Language of Guatemala", en Loanwords in the World's Languages: A Comparative Handbook. Martin Haspelmath, Uri Tadmor (eds.). Berlín: Walter de Gruyter GmbH and Co.; pp. 873-896.

Data recebimento: 05/11/2014.

Data aceite: $20 / 11 / 2014$. 